\title{
Identification of detailed behaviour of Flexible Manufacturing Cell using Hierarchical Timed Coloured Petri Nets
}

\author{
Sanjib Kumar Saren ${ }^{1, *}$, Florin Blaga ${ }^{2}$, and Tiberiu Vesselenyi ${ }^{1}$ \\ ${ }^{1}$ Mechatronics Department, University of Oradea, Romania \\ ${ }^{2}$ Industrial Engineering Department, University of Oradea, Romania
}

\begin{abstract}
The goal of this paper is focused on identifying the behavioural structure of a flexible manufacturing cell (FMC) using a model based on Coloured Timed Petri Nets and applying hierarchical techniques. In the paper we explain the modelling procedure of the manufacturing cell with single resource for different types of parts according to proposed decisions on places and transition in order to analyse the overall processing time in the cell. The designed model is based on a manufacturing cell from Faculty of Managerial and Technological Engineering, University of Oradea. In this present work, we present the behaviour of resource places, transitions and evaluate overall performance of the flexible manufacturing cell using CPNTools software.
\end{abstract}

\section{Introduction}

The useful nature of Petri Nets (PNs) resides in the power to identify the system behaviour in any kind of manufacturing model related to concurrent structures and asynchronies. Nowadays, the utilization of High Level Petri Nets in modern manufacturing sector for modelling purpose is rising. To establish a compound model using Petri Nets is a common subject now but the identification of the system behaviour in a particular manufacturing model is difficult. Coloured Petri Nets (CPN) provides system analysis and statistical analysis using state space analysis and helps to provide appropriate information about the behaviour in any kind of manufacturing model. The implementation of Hierarchy techniques in manufacturing system is not always necessary but for the design and modelling of complex manufacturing systems sometimes is very useful to build such models. To predict detailed behaviour of the cell or system the modelling and analysing is important. The design of hierarchical models with detailed behaviour with various levels is quite fashion in manufacturing system. Hierarchical Coloured Petri Net (HCPN) application in single model design with relation of sub-nets was first introduced by Huber [1]. Dynamic behaviour of automated manufacturing systems using coloured, timed, object

${ }^{*}$ Corresponding author: sanjibksaren@gmail.com 
oriented PNs was described by Wang [2]. Dynamic tool allocation and performance evaluation of the FMS model was explained by Chen in [3] using object-oriented Coloured Petri Nets. Viswanadham in [4] uses Coloured Petri Nets to model and invariant analysis of Flexible manufacturing cell. In [5] the application of extended Petri Nets in error free hierarchical FMS model with operation of each subsystem using single resource active cycle is described. Blaga in [6] explained the design procedure of a Flexible manufacturing cell (FMC) using CPNTools software. The author in [7] explained the application of CPNTools software to model mechatronics system with implementation of hierarchy and colour. In [8] the application of hierarchical coloured Petri Nets found in production line modelling is described and also the author explained the benefits of CPNTools to model the production system.

In this paper, we briefly explain the Hierarchy modelling techniques of flexible manufacturing cell using Coloured Timed Petri Nets in order to identify the detailed behaviour of the cell and also explain the design procedure of each sub model of the cell and the overall system performance.

\section{Flexible manufacturing cell configurations}

The proposed, modelled flexible manufacturing cell is operating at the Faculty of Managerial and Technological Engineering, University of Oradea shown in Figure 1. The Flexible manufacturing cell contains one CNC machining centre TMA 55 OP based on a FANUC control system, two ABB IRB 1600 robots equipped with Schunk pneumatic gripping devices, automatic tool changer with a tool capacity in the tool magazine of 40 tools, conveyor transfer system and separate storage area for specific raw material and finished parts and two pallets for transporting the parts in the cell.

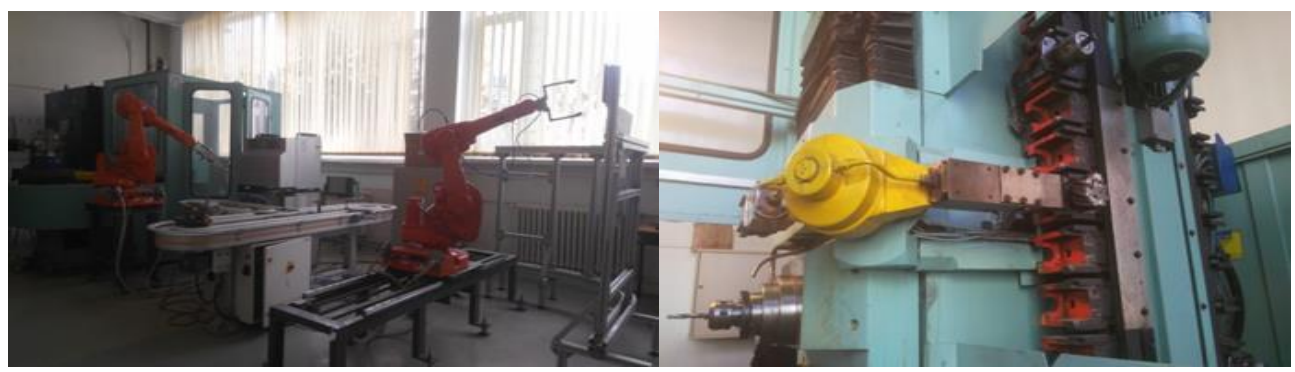

(a)

(b)

Fig. 1. (a) Flexible manufacturing cell from the Faculty of Managerial and Technological Engineering, University of Oradea. (b) Automatic tool changer system with tool magazine inside the machine.

\section{Proposed Hierarchical Flexible manufacturing cell model}

The concept of hierarchical timed Coloured Petri Nets (HTCPN) provides an extended representation of the developed model with colour, time and untimed, hierarchy, priority in transitions etc. Coloured Petri Nets provides a pure graphical model with advanced modelling facilities using hierarchical techniques. CPN Petri nets can be transformed to hierarchical PN using subnet of the model it helps the system to be more transparent with tabular structure which analyses the system more effectively and present the model more detailed in order to better be understand. In this proposed model, we used CPN Tools to construct a single machine cell model with colour, time and hierarchy. The base model is 
constructed with storage area for raw parts, for loading and unloading purpose we consider two robots, two pallets are considered for transport of raw parts and finished parts through the conveyor and a single machine for machining purpose. The resources are explained in the base model with mention of the places and each place contains meaningful parameters. CPNTools provides the means to construct the hierarchy model. In this cell we developed the base model with three sub models which are respectively: (a) part arrival sub model (b)

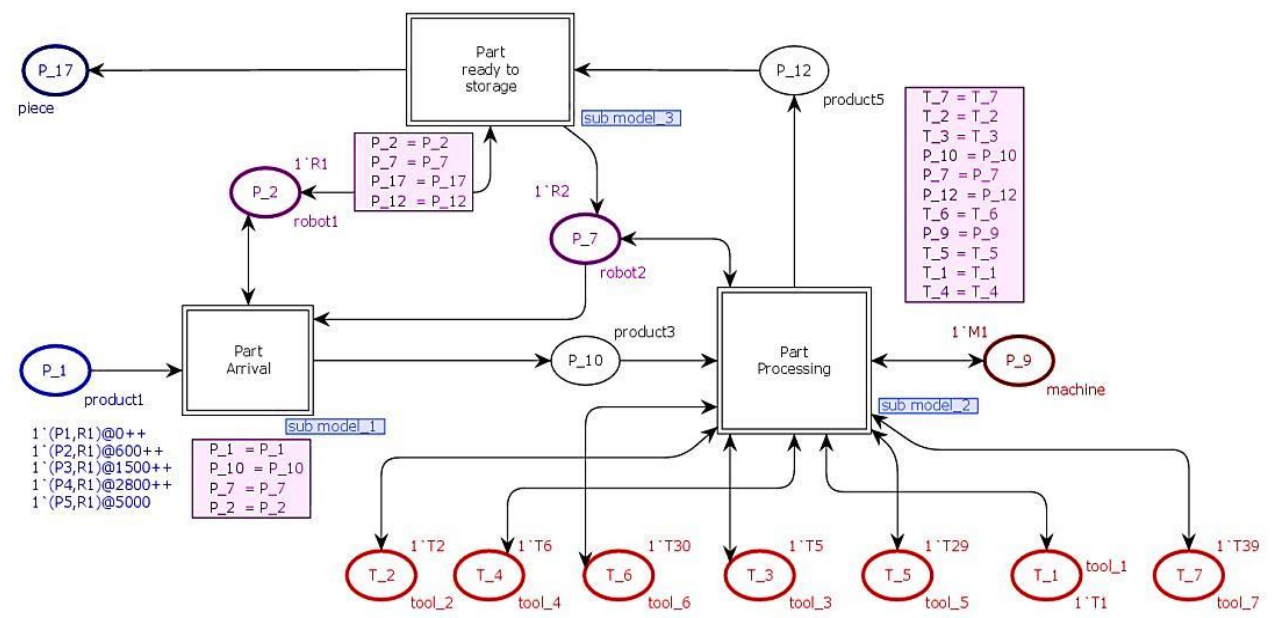

part processing sub model (c) Part complete sub model.

Fig. 2. Hierarchical structure of the flexible manufacturing cell.

Here, we define the colour set for the cell: (1) colset piece = with P1|P2|P3|P4|P5 timed; (2) colset machine = with M1 timed; (3) colset robot1= with R1 timed; (4) colset robot2= with R2 timed; (5) colset pallet1= with B1 timed; (6) colset pallet2= with B2 timed; (7) colset tool_1= with T1; (8) colset tool_2= with T2; (9) colset tool_3=with T5; (10) colset tool_4= with T6; (11) colset tool_5= with T29; (12) colset tool_ $\overline{6}=$ with T30; (13) colset tool_ $7=$ with T39. In figure 1 the hierarchical model contains the resource places and blocks of the sub model. In this hierarchical flexible cell, the places and their interpretation play significant role to understand the design of the cell. Place $P_{-} 1$ is Storage area for raw parts which have 5 colour token marking with 1 ( $(\mathrm{P} 1, \mathrm{R} \overline{1})++$ $1^{`}(\mathrm{P} 2, \mathrm{R} 1)++11^{\prime}(\mathrm{P} 3, \mathrm{R} 1)++1{ }^{\prime}(\mathrm{P} 4, \mathrm{R} 1)++1^{`}(\mathrm{P} 5, \mathrm{R} 1)++$, place $\mathrm{P} 22$ and $\mathrm{P} 7$ defines the robots used for loading and unloading purpose both have one colour token and the marking is 1'R1 and 1'R2, place P_9 defines the CNC machine which have one colour token carry marking value $1{ }^{\prime} \mathrm{M} 1$. All the tools have colour token value one and the places are respectively, T_1, T_2, T_3, T_4, T_5, T_6 and T_7. The marking are for the tools 1'T1, 1`T2, 1`T5, 1`T6, 1 'T29, 1'T30, $1^{`}$ T39. Other place in the model P_10 defines the connection between R1 and M1, P_12 define the connection between R2 and M1 and P_17 defines the final storage area for the finished parts.

\subsection{Sub-models of the cell}

In this proposed hierarchical flexible cell, arrival time is considered in resource place $\mathrm{P}_{-} 1$, for all five different parts mentioned in figure 3 . The part arrival time for $\mathrm{P} 1=0 \mathrm{sec}, \mathrm{P} 2=$ $600 \mathrm{sec}$, for $\mathrm{P} 3=1500 \mathrm{sec}, \mathrm{P} 4=2800 \mathrm{sec}$, for $\mathrm{P} 5=5000 \mathrm{sec}$ in the cell. All the parts are processed in the $\mathrm{CNC}$ machine and the observed time for each part is collected during experiment. The observed time according to parts are $\mathrm{P} 1=336 \mathrm{sec} ; \mathrm{P} 2=458 \mathrm{sec}$; $\mathrm{P} 3=$ $1158 \mathrm{sec} ; \mathrm{P} 4=2160 \mathrm{sec}$ and P5 = $2460 \mathrm{sec}$. 
Part arrival sub model can be described as follows: In part arrival section place P_1 contains arrival time for each part. In figure 3 , we mentioned the arrival time decision for five different parts in the cell such as $\mathrm{P}_{1} 1$ with $1^{\prime}(\mathrm{P} 1, \mathrm{R} 1) @ 0++1{ }^{`}(\mathrm{P} 2, \mathrm{R} 1)$ @600++1`(P3,R1)@1500++1`(P4,R1)@2800++1'(P5,R1)@5000. Five Colours token appears in the resource place which describes the availability of raw part in storage area. The transitions T1, T2, T3 and T4 contain delay time @+30 seconds to create connection between robot1, pallet1 and robot2 in the sub-model. In Table 2 and 3, we mention related transitions and places for the sub model. In this, sub-model robotl takes parts from storage area according to arrival time and unload of the part in pallet1 for transport to the next section. Robot1 is referred as an input/output function in this sub-model. Place P_1 and robot2 work as an input function and place $\mathrm{P}_{-} 10$ as an output function in the sub-model. The token value represents the status of the resource places such as in this sub-model place P_1 contain token value 5 which represent the number of parts in resource place and P_4

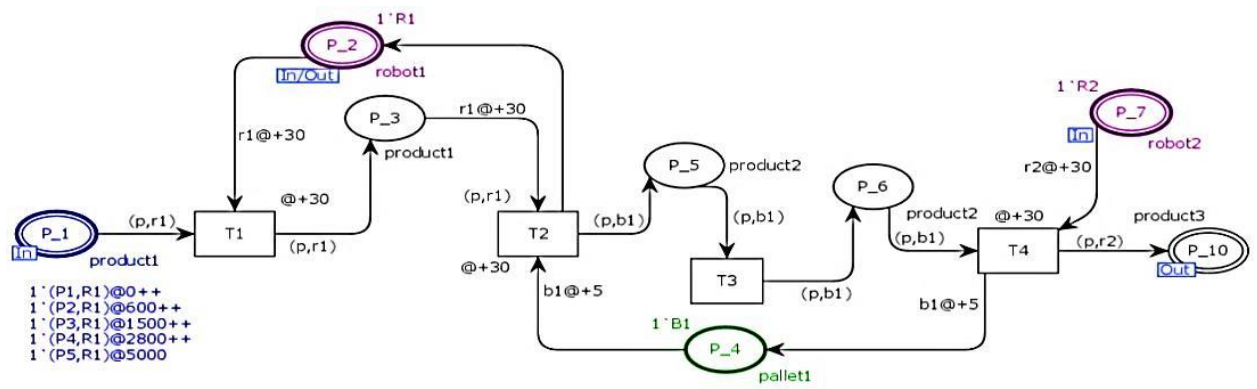

present the number of pallet with token value 1 .

Fig. 3. Sub model structure of part arrival for FMC.

In the part processing sub model: the selection of appropriate tool for processing parts is necessary. To construct this section each tool are represented in different resource places, such as 1`T1, 1`T2, 1`T5, 1`T6, 1`T29, 1`T30 and 1`T39.

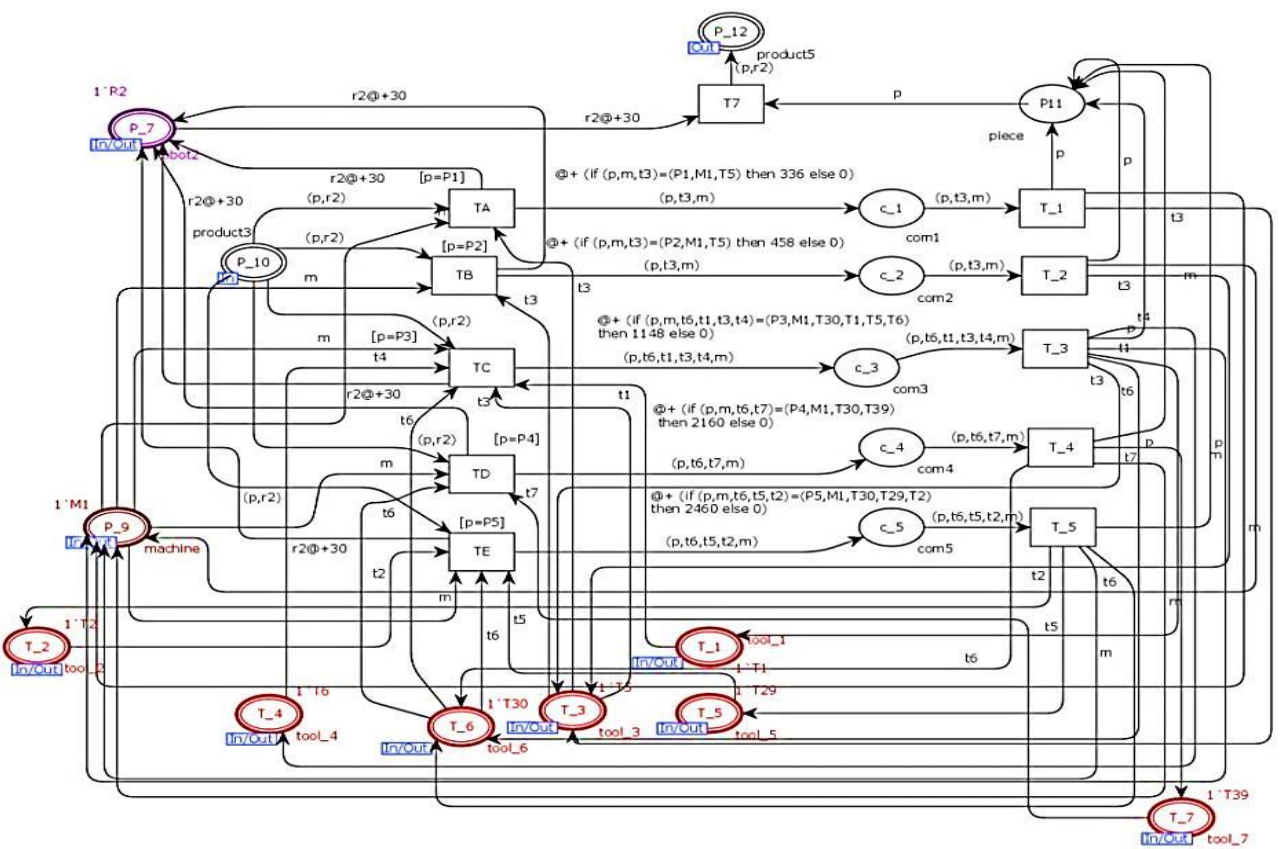


Fig. 4. Sub model structure of part processing for FMC.

The tools are used for different types of operations in the machine. The token value for the tools denoted 1 means only one tool is available in the resource places. The machine, robot 2 and all seven tools are defined as an input and output function in this sub model. During design in each transition we define a guard function to identify the exact connection between tool and part. In transition TA, the guard function [p=P1] is activated when information is carried by the arc from place to transitions. So, each transition is designed in such a manner that it can identify the exact part for processing in the machine using the appropriate tools. Also, in this section we have to establish a connection between place and transitions, in the arc we defined variables according to tool and parts. If we closely look to the design of this section, we observe that the processing time is defined in each transition. Each transition defines part processing time for each different part. The processing time is defined for $\mathrm{P} 1=336 \mathrm{sec}, \mathrm{P} 2=458 \mathrm{sec}, \mathrm{P} 3=1158 \mathrm{sec}, \mathrm{P} 4=2160 \mathrm{sec}$ and $\mathrm{P} 5=2460 \mathrm{sec}$ respectively. Each part is processed in the machine using appropriate place such as place c_1 for P1, c_2 for P2, c_3 for P3, c_4 for P4 and c_5 for P5. The manufacturing cell is based on single machine so each time only one transition is activated during operation in the cell. The part storage sub model is defined as followed: place P_2 defined as an input/output function and $P_{-} 12$ defined as an input function in the sub model. $P_{-} 7$ and $P_{-} 17$ are output functions in this sub model. Pallet 2 with resource place $\mathrm{P}_{-} 14$ contain token valued 1 with marking 1 'B2. This section describes the operation when the part is completed robot 2 load the part in pallet 2 and transfer the part near robot 1 and the robot 1 unload the part from pallet2 and store the finished part in the storage area. If we closely look at the model we can observe the delay of $30 \mathrm{sec}$ for robot 1 and robot 2 for loading and unloading purpose.

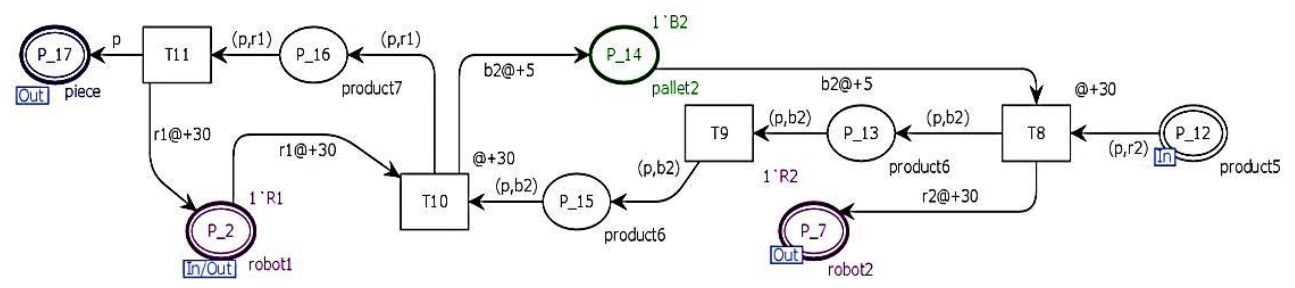

Fig. 5. Sub model structure of end section for FMC.

\section{Simulation results}

In this flexible manufacturing cell, we are processing five different parts in a single machine according with part arrival time decisions. The part will be released from place $\mathrm{P}_{-} 1$ with a specific time. During simulation it is identified that robotl take the part from resource place and load the part in pallet1 to transfer it through the conveyor. In a specific time later the robot2 takes the part from pallet1 and put it inside the $\mathrm{CNC}$ machine, when the part is processed the sub model is activated and the appropriate tool is selected from resource places for the defined part in the cell. Such as, for example, in transition TA, tool_3 is activated for processing part1 in the machine. During simulation, it's identified that the colour token is removed from resource places and performs the operation according to firing on transitions. The part processing is following the arrival time and the part processing time in the cell. After completion of the part robot2 unloads the part from the machine and loads it in pallet2 which carry the part through the conveyor and when robot1 is free it takes the part from pallet 2 and loads it in the final storage area. Coloured Petri Nets helps to define simulation process by moving colour token from one place to another. In figure 6 , we define the simulation observation after processing all the parts. The cell is modelled using time units so; the time will appear in the mentioned places. 
The total time appears in place $\mathrm{P}_{-} 17$ with marking value 1’P1@486+++1'P2@1208+++1'P3@2798+++1'P4@5110+++1'P5@7630. Also, in place P_2, P_7 and P_9 the time appears after simulation respectively $7660 \mathrm{sec}, 7630 \mathrm{sec}$ after 55 steps. CPNTools provides the number of steps to justify the system identification and provide possible information according to token values and specific time mentioned in resource places and transitions.

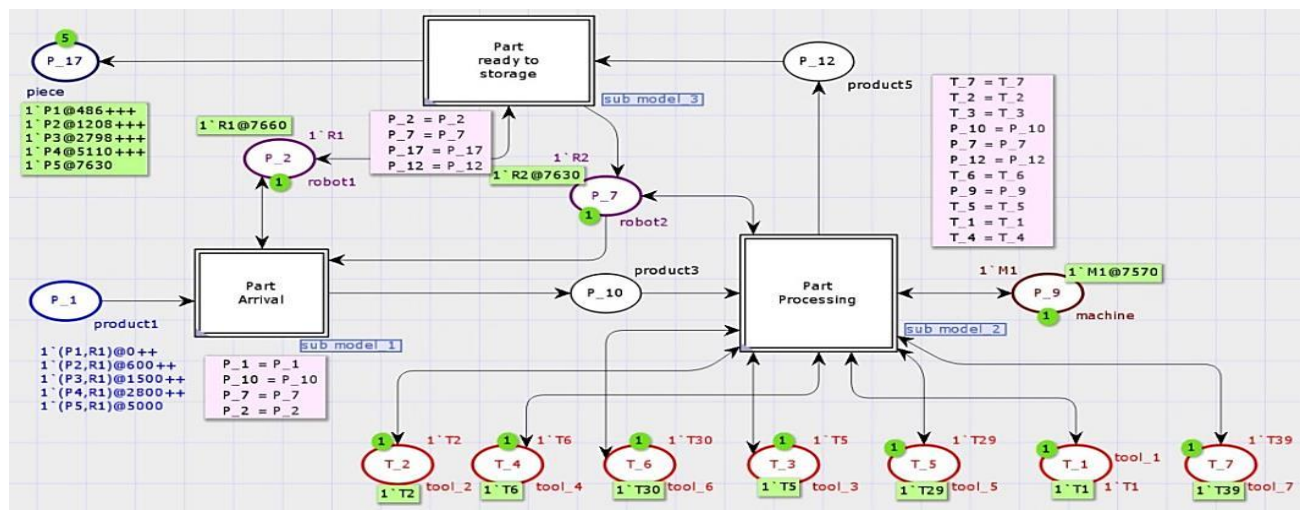

Fig. 6. Final time appears in base model after simulation runs.

\section{Conclusions}

In this paper we described the modelling techniques and simulation experiment of a flexible manufacturing cell using CPNTools software implement of hierarchical formalism. The contribution of this work was the modelling of flexible cell and the identification of the behaviour of each sub model by simulation of the cell. Each sub model carry significant characteristic to identify the status of the resource places. The implementation of time in hierarchical model provides more realistic results compared with normal Colour Petri Nets. Here, in this cell, the total time observed from the simulation which helps to understand the value for real system.

The paper published has been sponsored under the Erasmus mundus partnership program agreement vide number 2014-0855/001-001 coordinated by and between University of Oradea and City University of London Under Action Plan 2 for the year 2015-2018.

\section{References}

1. P. Huber, K. Jensen, R.M. Shapiro, In: Rozenberg G. (eds) Advances in Petri Nets, Lecture Notes in Computer Science, Springer-Verlag (1990).

2. L. C. Wang, S. Y. Wu, Computers and Industrial Engineering, 2, 34, (1998).

3. J. Chen, F.F. Chen, 2, 21, (2003).

4. N. Viswanadham, Y. Narahari, Proc. IEEE Int. Conf. Robot. Automat, 4, (1987).

5. K. P. Valavanis, IEEE Transactions on Systems, Man, and Cybernetics, 1, 20, (1990).

6. F. Blaga, I. Stanasel, A. Pop, V. Hule and T. Buidos, Annals of the Oradea university, Fascicle of Management and Technological Engineering, 1, 4 (2014).

7. Jadlovský. J, Ilkovič. J, Procedia Engineering, 48, (2012).

8. Eloundou. J, Baudry. D, Bensrhair. A, Xth International Conference on Integrated Design and Production, CPI (2015). 